\title{
Movement-Accuracy Control in Tetraparetic Cerebral Palsy: Effects of Removing Visual Information of the Moving Limb
}

\author{
Dominique van Roon, Bert Steenbergen, and \\ Ruud G.J. Meulenbroek
}

\begin{abstract}
People with cerebral palsy $(\mathrm{CP})$ are known to rely heavily on visual guidance when making targeted upper-limb movements. In the present study, we examined whether being able to visually monitor the moving limb forms a precondition for people with $\mathrm{CP}$ to make accurate upper-limb movements. Eight participants with tetraparetic $\mathrm{CP}$ and eight controls were asked to produce large-amplitude, straight-line drawing movements on a digitizing tablet. In half the trials, vision of the moving limb was blocked. Accuracy constraints were manipulated by varying the width of the target and by imposing a maximum width of the movement path. Surprisingly, when vision was blocked movement accuracy was comparable in the two groups. Thus, people with tetraparetic $\mathrm{CP}$ do not strictly require constant vision of their moving limb to make accurate upper-limb movements. They compensated for the lack of visual information, however, by prolonging movement time. Using a high pen force proved a general strategic adaptation, possibly to filter out unwanted noise from the motor system or to enhance proprioceptive input.
\end{abstract}

Key Words: motor control, CP, visual guidance, accuracy, proprioception

It is known that individuals with cerebral palsy $(\mathrm{CP})$ have difficulties in performing accurate upper limb movements (Brown et al., 1989; Steenbergen, Meulenbroek, \& Rosenbaum, 2004; Van Thiel, Meulenbroek, Hulstijn, \& Steenbergen, 2000). This can be a consequence of disordered motor coordination (Barnes, McLellan, \& Sutton, 1994; Filloux, 1996; Lance, 1980) or impaired proprioception (Cooper, Majnemer, Rosenblatt, \& Birnbaum, 1995; Opila-Lehman, Short, \& Trombly, 1985) that are associated with $\mathrm{CP}$, causing the motion to deviate from the centrally programmed trajectory. As a result, there is an increased need for the movements to be corrected on-line (cf., Steenbergen \& Van der Kamp, 2004). Corrective movements can be made on the basis of visual and/or proprioceptive feedback. As the proprioception of people with CP is known to be impaired, however, (Cooper et al., 1995; Opila-Lehman et al., 1985), the use of visual feedback might become the

The authors are with the Nijmegen Institute for Cognition and Information, Radboud University, P.O. Box 9104, 6500 HE Nijmegen, the Netherlands. 
dominant strategy to keep the accuracy of moving at an acceptable level. Indeed, it has frequently been observed that people with CP rely more on visual guidance (or focal vision) of the moving hand during upper limb movements as compared to control participants (e.g., Steenbergen, 2000, p.81; Steenbergen \& Van der Kamp, 2004; Trombly, 1992). Furthermore, Wann (1991) showed that visual-proprioceptive mapping is impaired in people with severe CP. Individuals with CP encounter problems when visual and proprioceptive information need to be encoded into a common egocentric frame of reference. This deficit can cause impaired motor control, and thus impaired accuracy, when a movement has to be made towards a visible target without visual information of the moving limb. The increased need for visual control of movements, as compared to controls, has been shown for several patient groups, such as Parkinson's disease (Adamovich, Berkinblit, Hening, Sage, \& Poizner, 2001; Klockgether \& Dichgans, 1994), hemiparetic stroke (Pryde, Roy, Bryden, Kalbfleisch, \& MacKinnon, 1998), cerebellar disorders (Cody, Lovgreen, \& Schady, 1993), and attention deficit hyperactivity disorder (ADHD; Eliasson, Rösblad, \& Forssberg, 2004). To date, however, the role of visual guidance for accuracy control has not been explicitly examined in people with CP.

One possible way to study visual control is to register eye movements. It was previously shown that healthy people usually fixate their eyes on the target, either before actual limb movement or very early in the limb trajectory (Abrams, Meyer, \& Kornblum, 1990; Bekkering, Adam, Van den Aarssen, Kingma, \& Whiting, 1995; Neggers \& Bekkering, 1999; Vercher, Magenes, Prablanc, \& Gauthier, 1994). As a consequence, the moving hand is primarily seen in peripheral vision during the larger part of the movement. This peripheral visual information is used either on-line to enable corrections during the movement, or off-line to improve the programming of the subsequent movements (e.g., Abahnini \& Proteau, 1999; Bédard \& Proteau, 2004; Khan, Lawrence, Franks, \& Buckolz, 2004). If people with CP use focal vision of their moving hand to control their movements, the time they direct their gaze at their moving hand should be longer than the time they look at the target. Importantly, although an eye movement study can reveal whether people with $\mathrm{CP}$ use visual guidance of their moving limb more than controls, it cannot be determined whether they really need that guidance to be able to move accurately.

To answer that question, we experimentally manipulated the availability of visual information of the moving hand when participants had to move a pen on a digitizing tablet towards a goal located at a 32-cm distance from the starting position. In half of the trials, vision of the moving arm was blocked by a screen. In the other half, full vision of the arm was available. Accuracy constraints were manipulated both by varying the width of the target and by imposing a maximum width on the movement path by adding two lines between the starting and target area between which the drawing traces had to be made.

Under the assumption that visual guidance of the moving limb is a prerequisite for accuracy containment in $\mathrm{CP}$, we hypothesized that blocking vision of the moving limb would more negatively affect accuracy of moving in participants with CP than in controls. It is also possible, however, that people with CP can use compensatory strategies to cope with the loss of vision.

The first strategy participants might use is an increase in pen force. Results of previous studies suggest that an increase in pen force enhances proprioceptive input from the moving limb (Reinders-Messelink et al., 2001). Furthermore, it could serve 
as a filter for unwanted neuromotor noise (Van Galen \& Van Huygevoort, 2000; Van Gemmert \& Van Galen, 1997). Based on these studies, we expected a higher pen force in the participants with CP. In addition, if people with CP would use this strategy, we expected a higher pen force when visual feedback of the moving limb would be removed, and when accuracy constraints of the task would be increased (moving to a small target and between two lines at a small distance from each other; see also Gribble, Mullin, Cothros, \& Mattar, 2003).

The second strategy that might be employed to compensate for the lack of visual information is to make slower movements (Berthier, Clifton, Gullapalli, McCall, \& Robin, 1996; Churchill, Hopkins, Ronnqvist, \& Vogt, 2000; Smyth, Peacock, \& Katamba, 2004; but see also Bédard \& Proteau, 2003). According to the signal-dependent noise theory (Harris \& Wolpert, 1998; Van Beers, Baraduc, $\&$ Wolpert, 2002), a slower movement requires a smaller control signal (motor command) than a faster movement, and a smaller control signal is presumed to be less noisy. Also, with slower movements, more time is available to process proprioceptive feedback and to correct the movement based on that information. It might be possible that people with $\mathrm{CP}$ benefit from that extra time when visual feedback is not available. If people with CP use this strategy, we expect their movements to be slower in conditions where vision of the moving limb is absent, and when the accuracy constraints of the task are increased.

In sum, our two main research questions were: (a) Do people with CP really need visual guidance to be able to move accurately?; and (b) When visual information of the moving limb is not available, do people with CP employ compensatory strategies, e.g., increasing pen force or making slower movements, to enable them to move accurately?

\section{Methods}

\section{Participants}

Eight individuals being diagnosed with having mild spastic tetraparesis as a consequence of $\mathrm{CP}$ (mean age, 18 years, $S D$, 2 years, 1 month; range, 15 years, 1 month to 20 years, 6 months), and 8 control participants with no known history of neurological disorders (mean age, 22 years, 5 months; $S D, 2$ years, 11 months; range, 19 years, 2 months to 28 years, 5 months) voluntarily participated in the experiment. At the time of testing, all participants with $\mathrm{CP}$ were students at the Werkenrode Institute (Groesbeek, The Netherlands), where they followed an adapted educational program. They all had sufficient physical and cognitive abilities to perform the task under study, and had normal or corrected to normal vision. None suffered from apraxia or neglect. Six tetraparetic participants sat unstrapped in a wheelchair from which they performed their daily activities. The other two were able to walk independently. Gross dexterity of the tetraparetic participants was assessed with the Box and Block test (Mathiowetz, Volland, Kashman, \& Weber, 1985) according to the instructions in the test manual. Active and passive proprioception measures were taken for each participant individually to assess the level of proprioceptive impairment. See Table 1 for the individual scores on these tests, and for additional participant information. Participants gave signed consent prior to data collection. This study was approved by the local ethics committee and performed in accordance with the ethical standards laid down in the 1964 Declaration of Helsinki. 


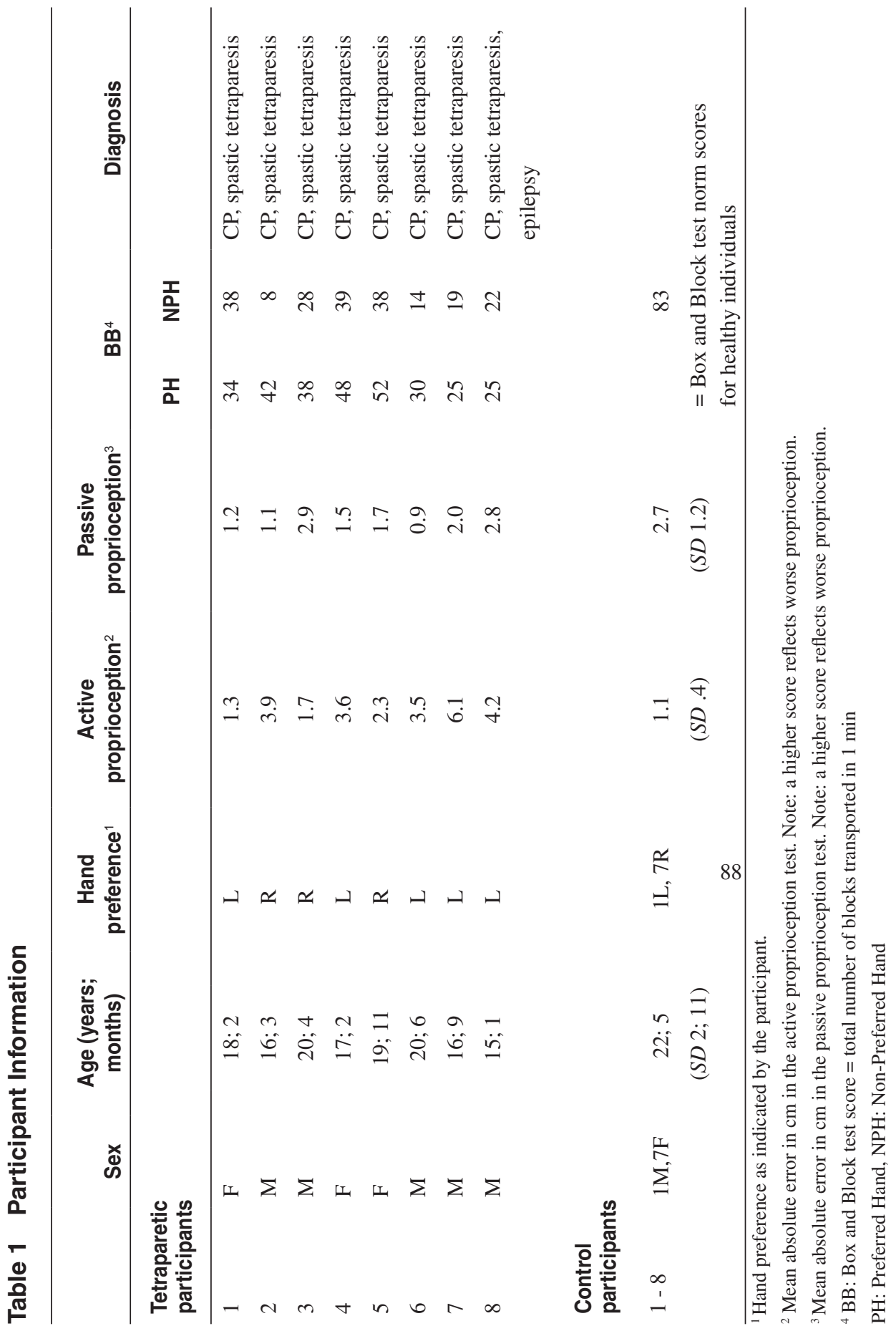




\section{Passive Proprioception Test (Kinesthetic Recall)}

Experimental Setup. A custom made apparatus was used, consisting of a hard plastic ball (diameter, $5 \mathrm{~cm}$ ) that could run along a rail. A ruler was attached at the side of the rail. The rail was placed on the table in the midsagittal plane directly in front of the participant. Vision of the participant's limb was blocked by means of an opaque black sheet (see Figure 1).

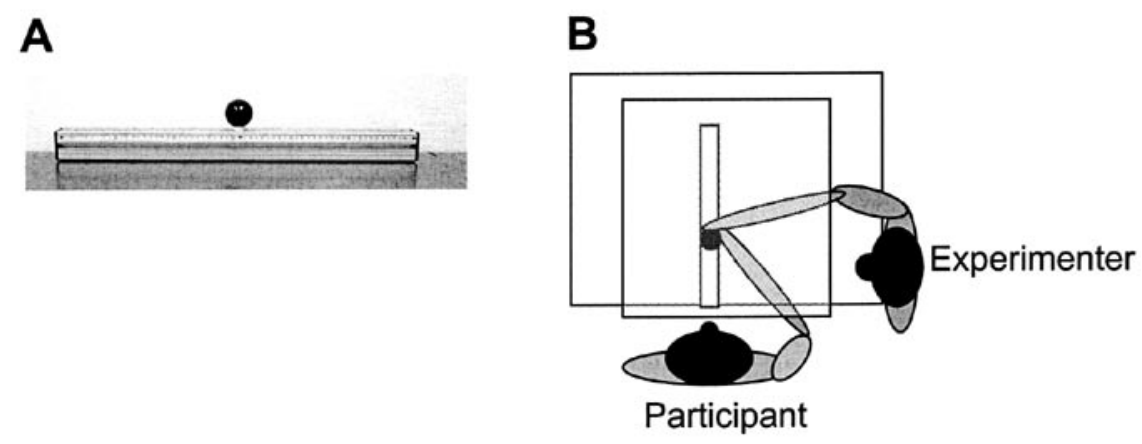

Figure 1-The passive proprioception test: (A) the apparatus used, and (B) top view of the experimental setup. Vision of the moving limb was blocked by an opaque black sheet.

Procedure. Participants were instructed to hold the plastic ball with their preferred hand throughout the performance of the task. The preferred hand was the hand used for writing. First, the experimenter slowly moved the ball forward along the rail to a predetermined position. Next, the participant was asked to remember that exact position of the ball. Subsequently, the ball was moved back to the starting position by the experimenter. Finally, the experimenter slowly moved the ball again from the starting position along the rail and the participant had to indicate when the ball had reached the remembered position. This position was registered. Proprioception was tested at four distances $(7,14,21$, and $28 \mathrm{~cm}$ from the starting position). At each distance, the test was repeated four times. The order of trials was completely randomized. As measures of accuracy, we determined for each participant both the mean absolute error by calculating the mean absolute difference between the predetermined positions and the remembered positions, and the variability of the error by calculating the standard deviation of the signed errors across all trials.

\section{Active Proprioception Test}

Experimental Setup. An A3 WACOM digitizer (UD-1218-RE, sample frequency $170 \mathrm{~Hz}$, spatial precision $0.2 \mathrm{~mm}$ in the X-Y plane) was used, combined with an electronic pen with an inkless penpoint. The short side of the digitizer was placed parallel to the front edge of the table. We ensured that the writing surface was parallel to the tabletop. A cardboard box with a square hole at the front was placed on top of either the left or right half of the digitizer. A sheet of paper was placed on the digitizer, on which, on the visible side, horizontal lines were drawn at four distances (at 9, 16, 23, and $30 \mathrm{~cm}$ from the bottom of the sheet; see Figure 2). 

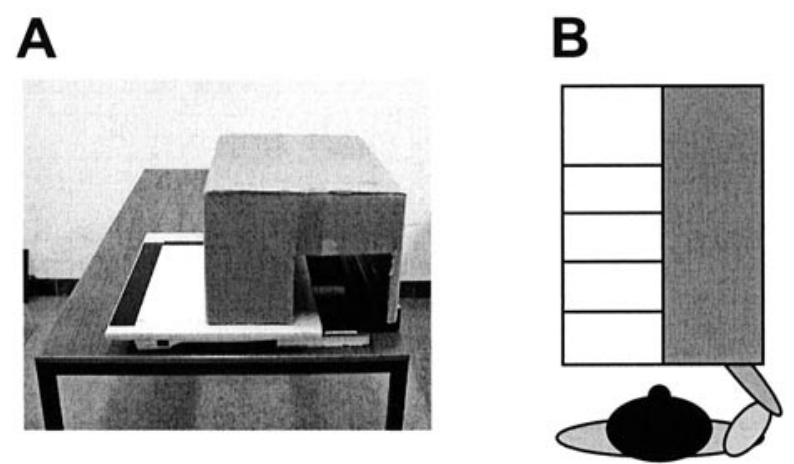

Figure 2-Photograph (A) and top view (B) of the experimental setup used in the active proprioception test.

Procedure. Participants held the pen in their preferred hand to draw a vertical line on the digitizer to a position corresponding to the position of one of the four lines. Because they had to draw underneath the box, they could not see their moving hand. The instruction was to stop drawing when the pen had reached the position of the horizontal line that the experimenter had instructed (line 1,2, 3, or 4). At each distance, the test was repeated four times and the order of trials was completely randomized. As in the passive proprioception test, we determined the mean absolute error and the variability of the error for each participant.

\section{Main Experiment}

Experimental Setup. An A3 WACOM digitizer (UD-1218-RE, sample frequency $170 \mathrm{~Hz}$, spatial precision $0.2 \mathrm{~mm}$ in the X-Y plane) was used, combined with an electronic pen with an inkless penpoint. Besides the 2D position of the pen on the surface of the digitizer, the axial pen force was registered. The digitizer was placed at the table directly in front of the participant. The short side of the digitizer paralleled the front edge of the table and the writing surface paralleled the tabletop. The starting circle, which was $5 \mathrm{~mm}$ in diameter, was drawn in the middle of the paper near the bottom so that the distance between the edge of the table and the starting position was $6 \mathrm{~cm}$. Two black rectangles were used as targets. The small target measured $5 \mathrm{~mm} \times 10 \mathrm{~mm}$ (width $\times$ depth). The large target measured 56 $\mathrm{mm} \times 10 \mathrm{~mm}$ (width $\times$ depth). The distance between the midpoint of the starting circle and the midpoint of the target was always $32 \mathrm{~cm}$ (see Figure 3, panel A). As a consequence, the indices of difficulty were 7 and 3.5 for the small and large targets, respectively. In half of the trials, two lines were present between the starting circle and the target. These lines were 5 and $56 \mathrm{~mm}$ apart for the small and large target, respectively (see Figure 3, panel A). In half of the trials, the availability of visual information of the moving limb was blocked by placing a hardboard screen above the digitizer (see Figure 3, panel B). The height and the depth of the screen were adjusted so that participants could only see their hand and the pentip at the start and end of the movement. The target remained visible throughout the task, allowing participants direct feedback about the endpoint accuracy. 

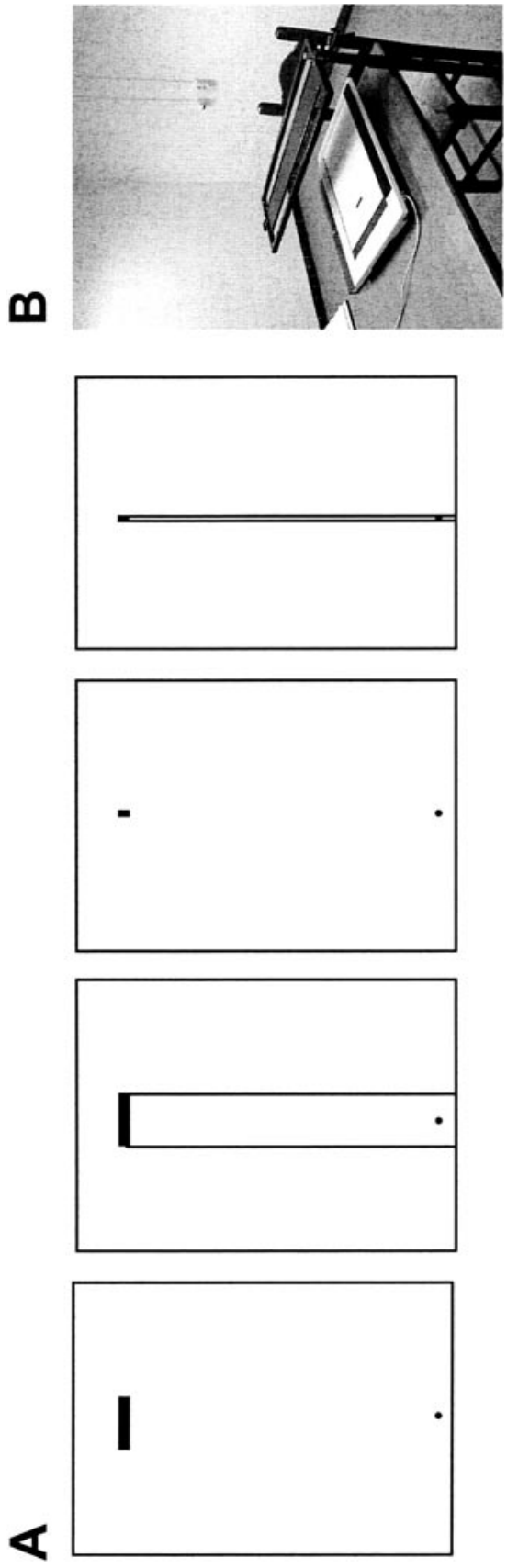

Figure 3-(A) The four accuracy conditions defined by target $(5 \mathrm{~mm} \times 10 \mathrm{~mm}$ or $56 \times$ $10 \mathrm{~mm}$ ) and lines (absent or present) used in the main experiment. The distance between the midpoints of starting circle and target is $32 \mathrm{~cm}$. (B) The experimental setup used in the main experiment. The digitizer was placed directly in front of the participant. Vision of the moving limb was blocked in half of the trials by an adjustable hardboard screen placed above the digitizer. 
Procedure. Participants were seated at a table on a wooden chair without armrests and with a high back. Each participant was able to reach the targets without moving the trunk. Participants were instructed to draw a line from the starting circle to the target rectangle as fast and as accurately as possible, holding the pen in their preferred hand. When lines were present, it was stressed that they had to draw the line between them. Participants had to keep their back against the back of the chair during the task to ensure that they could not see parts of the trajectory when the screen was placed above the digitizer. After a computer-generated beep ( $400 \mathrm{~Hz}, 500$ $\mathrm{ms})$ they began the movement. When the target was reached, another beep $(1,000$ $\mathrm{Hz}, 300 \mathrm{~ms}$ ) indicated that the participant had to place the pen in the starting circle again, before the start of the next trial. Each of the 8 conditions $(2$ target widths $x$ 2 line conditions [lines absent or present] $\times 2$ vision conditions [available or not]) was practiced two times. During the actual experiment, 16 blocks of 10 trials were carried out. The order of the blocks was ABBA-counterbalanced.

Data Analysis. The 2D positional data of the pen were filtered using a zero phase lag, second-order Butterworth filter with a cut-off frequency of $10 \mathrm{~Hz}$ and then differentiated to calculate movement velocity and acceleration. Semi-automatic segmentation routines were used to define the start and end of each movement. The start of the movement was defined as the moment at which the tangential velocity of the pen rose above $5 \mathrm{~mm} / \mathrm{s}$ for the first time and the pen was moving in a forward direction. The end of the movement was defined as the moment at which the tangential velocity finally fell below $5 \mathrm{~mm} / \mathrm{s}$.

The following dependent variables were calculated for each trial: movement time (MT), mean pen force, dysfluency, and accuracy of moving. The dysfluency of the movement was determined by the number of zero-crossings per second in the acceleration profile of the pen. As a measure of accuracy, we determined at several movement stages the mean absolute lateral deviation of the realized trajectory from the straight line between the starting point and the midpoint of the target. We divided each trajectory into 20 equal parts on the basis of the distance covered by the pen in the forward $(Y)$ direction. Subsequently, the $X$-coordinate of the position of the pen at the start of each of the parts and at the end of the movement (making 21 stages in total) was determined, followed by a calculation of the mean distance to the straight line. Another measure of accuracy was determined only in the conditions in which the participant had to move between 2 lines. It was defined as the time (expressed as percentage of MT) during which the pen was outside of these lines.

Statistical Analysis. The statistical procedures included one-tailed independent $t$-tests for comparisons between the tetraparetic and control groups, and repeated measures ANOVAs to compare the means of the dependent variables across the replications of each condition for each group separately. The design for the repeated measures ANOVAs consisted of three within-participant factors, namely target (large versus small), lines (absent or present), and vision (available or not available). Step-down analyses of statistically significant interactions were performed by means of contrasts. In addition, Spearman rank order correlation coefficients were calculated to explore the relationships between the measures of proprioception and performance in the main experiment. A one-tailed test of significance was used if we had a clear prediction about the direction of the correlation and a two-tailed test was used if this was not the case. An alpha level of .05 was used for all statistical tests. 


\section{Results}

\section{Passive and Active Proprioception}

The passive proprioception was worse in the tetraparetic group than in the control group. The mean absolute error in the passive proprioception test was $1.76 \mathrm{~cm}$ $(S D=0.75 \mathrm{~cm})$ for the tetraparetic participants and $1.13 \mathrm{~cm}(S D=0.38 \mathrm{~cm})$ for the controls, $t(14)=2.11, p=.027$. The mean variability of the error in the passive proprioception test was $2.00 \mathrm{~cm}(S D=0.54 \mathrm{~cm})$ for the tetraparetic participants and $1.38 \mathrm{~cm}(S D=0.37 \mathrm{~cm})$ for the controls, $t(14)=2.68, p=.009$.

While no differences were found between the groups with regard to the absolute error in the active proprioception test, the tetraparetic participants did show larger error variability than the controls $[2.22 \mathrm{~cm}(S D=0.90 \mathrm{~cm})$ and $1.20 \mathrm{~cm}(S D=0.54$ $\mathrm{cm}]$, respectively; $t(14)=2.73, p=.008$.

\section{Accuracy of Moving}

In Figure 4, raw data of a control participant and a tetraparetic participant are depicted. Ten successive movement trajectories (left panels) and the accompanying velocity profiles (right panels) are displayed for the "large target-no lines condition" (upper four panels) and the "small target-lines condition" (lower four panels), both with and without vision. In these typical examples, it can be observed that no extreme sideward drift was present in the trajectories, even when vision of the limb was not available. The task was accurately performed by both participants.

Lateral Deviation from Straight Line Between Starting Point and Midpoint of Target. First, it must be noted that the absolute deviation from the straight line between starting point and midpoint of the target was rather small (Figure 5). On average, this deviation never exceeded $1 \mathrm{~cm}$, while the participants had to cover a distance of $32 \mathrm{~cm}$ with the pen. Also, participants were able to diminish the lateral deviation after it had reached a maximum, even in conditions in which vision was blocked.

To determine whether the groups differed with respect to the mean absolute lateral deviation, we compared the groups using one-tailed $t$-tests at each of the 21 stages $(0-100 \%$ of the distance covered by the pen in the forward direction, in steps of 5\%), for the vision and no vision conditions separately. When vision of the moving limb was available, tetraparetic participants showed larger absolute deviations than the control participants throughout the larger part of the movement (mean difference: $0.2 \mathrm{~cm} ; p<.05)$. Only near and at the end of the movement $(95 \%$ and $100 \%$ ), no significant group effect was found. When vision was blocked, group differences were only found immediately after the start of the movement $(0-10 \%$; mean difference: $0.07 \mathrm{~cm} ; p<.05)$, and near and at the end of the movement (85-100\%; mean difference: $0.16 \mathrm{~cm} ; p<.05)$.

To determine the effects of blocking vision on the lateral deviation, we statistically analyzed the effects on the maximum deviation. A significant target $\times$ lines $\times$ vision interaction was found in both the tetraparetic group, $F(1,7)=5.62, p=$ .05 , and the control group, $F(1,7)=7.53, p=.029$. In the tetraparetic group, the 

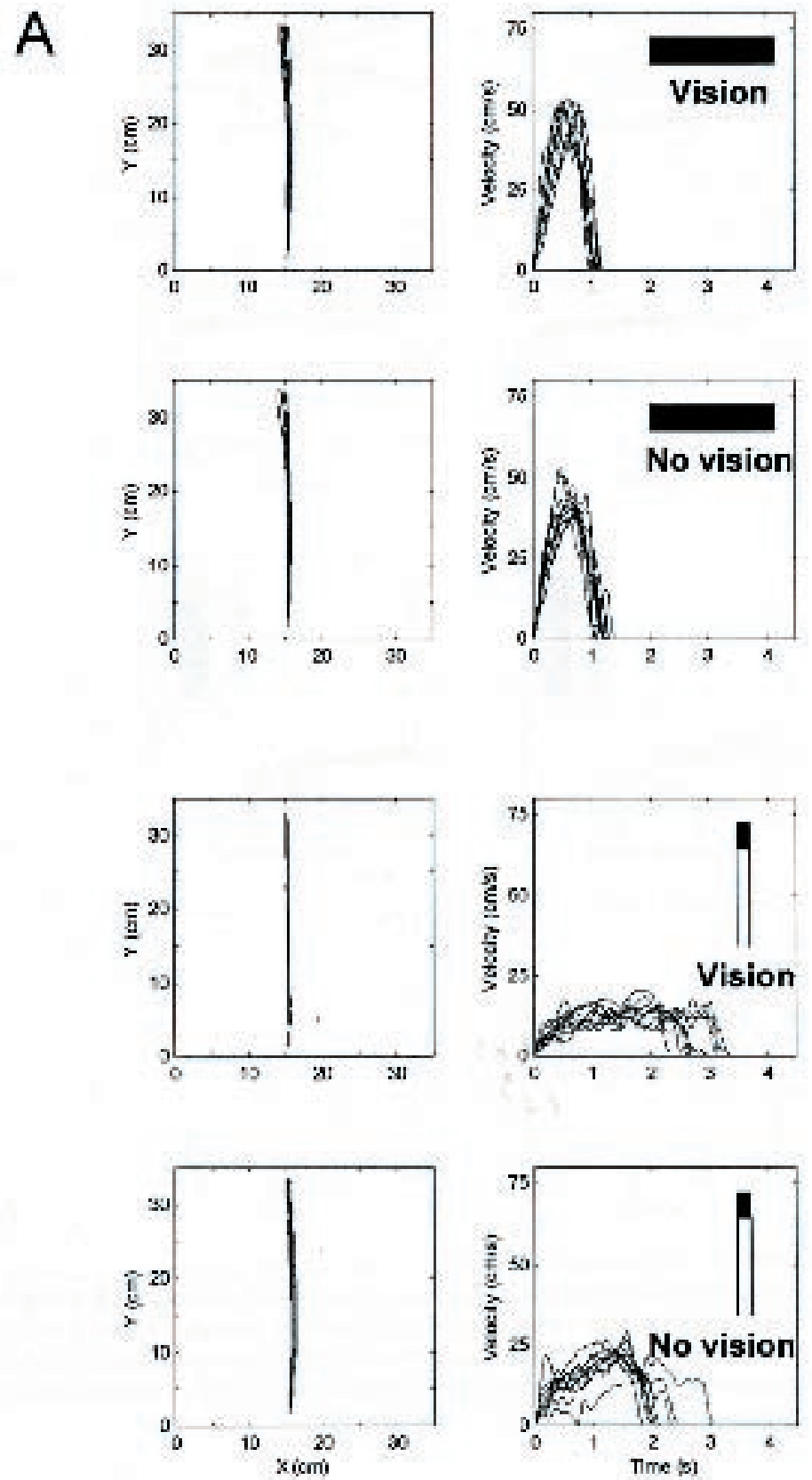

Figure 4-Raw data. Ten subsequent movement trajectories (left panels) and the accompanying velocity profiles (right panels) are depicted for the "large target-no lines condition" (upper four panels) and the "small target-lines condition" (lower four panels), both with and without vision. A: Data from a control participant. B: Data from a tetraparetic participant. 
B
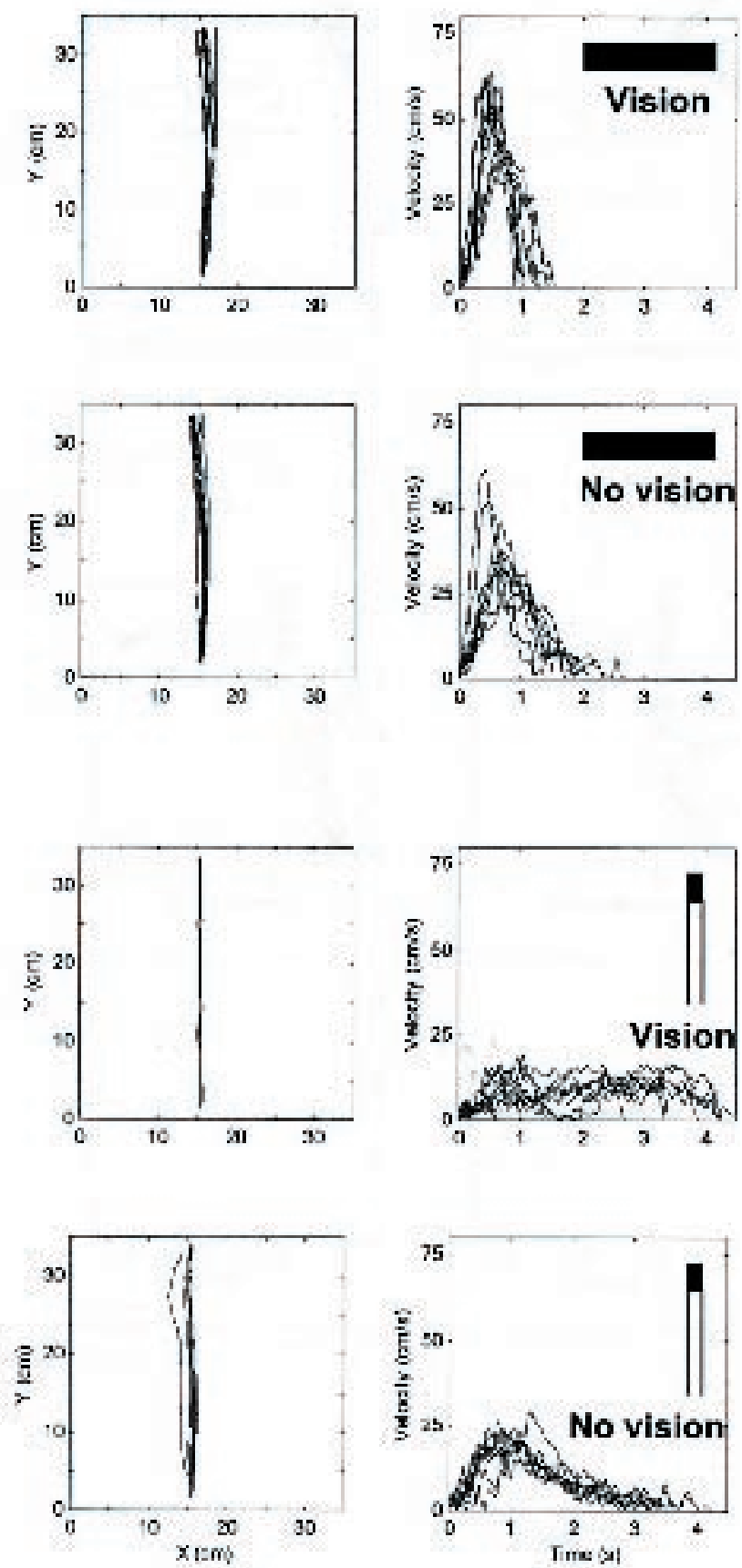

Figure 4-(continued) 
effect of blocking vision was only significant in the most difficult condition (small target - lines present, $p=.001$ ). In the control group, this effect was significant in both conditions in which lines were present (large target - lines present, $p=.042$; small target - lines present, $p=.005$ ). As noted above, the differences, although statistically significant, were negligible.

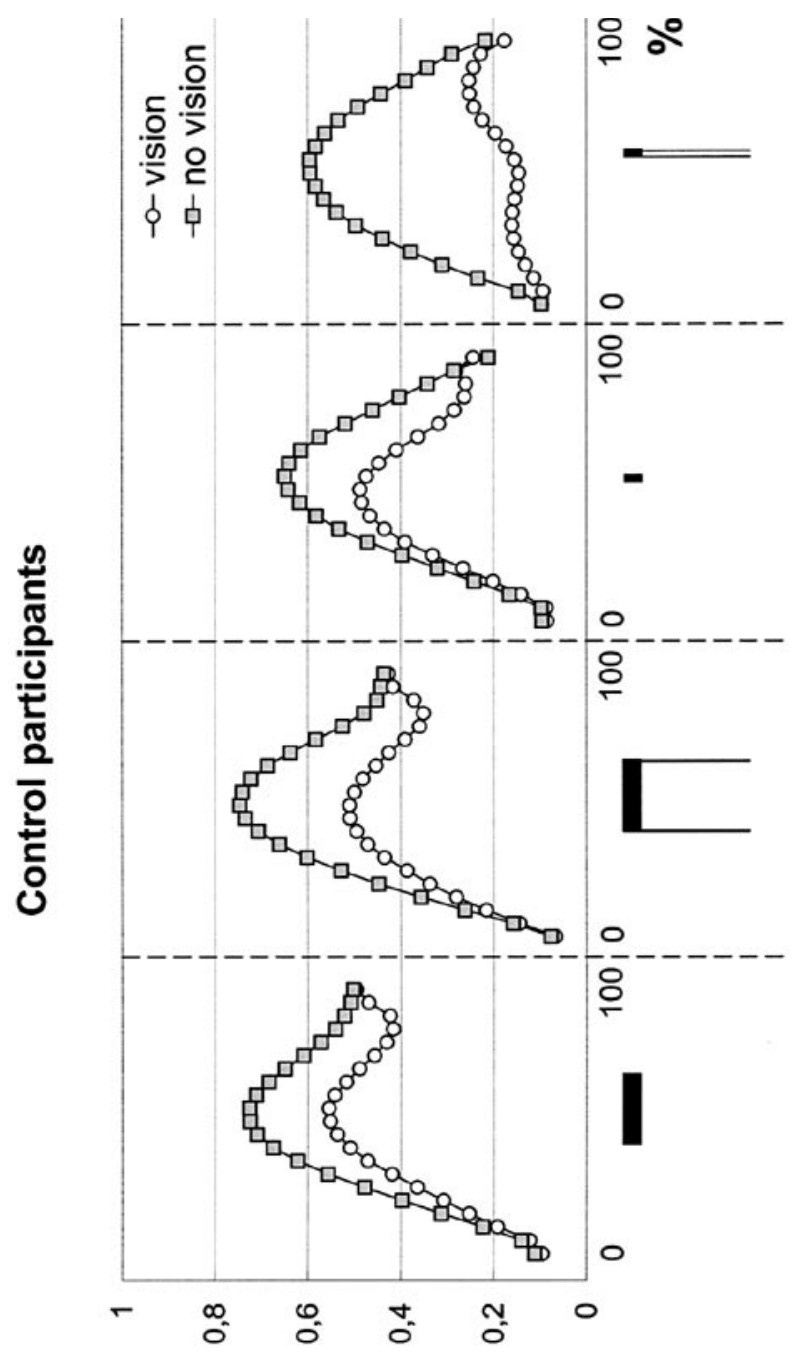

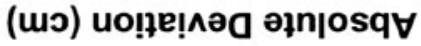

Figure 5-The mean absolute lateral deviation $(\mathrm{cm})$ of the pen from the straight vertical line between starting point and midpoint of the target for the control and tetraparetic participants as a function of target, lines, and vision. The deviation was calculated at $5 \%$ intervals of the distance in the forward $(Y-)$ direction between start and endpoint. 


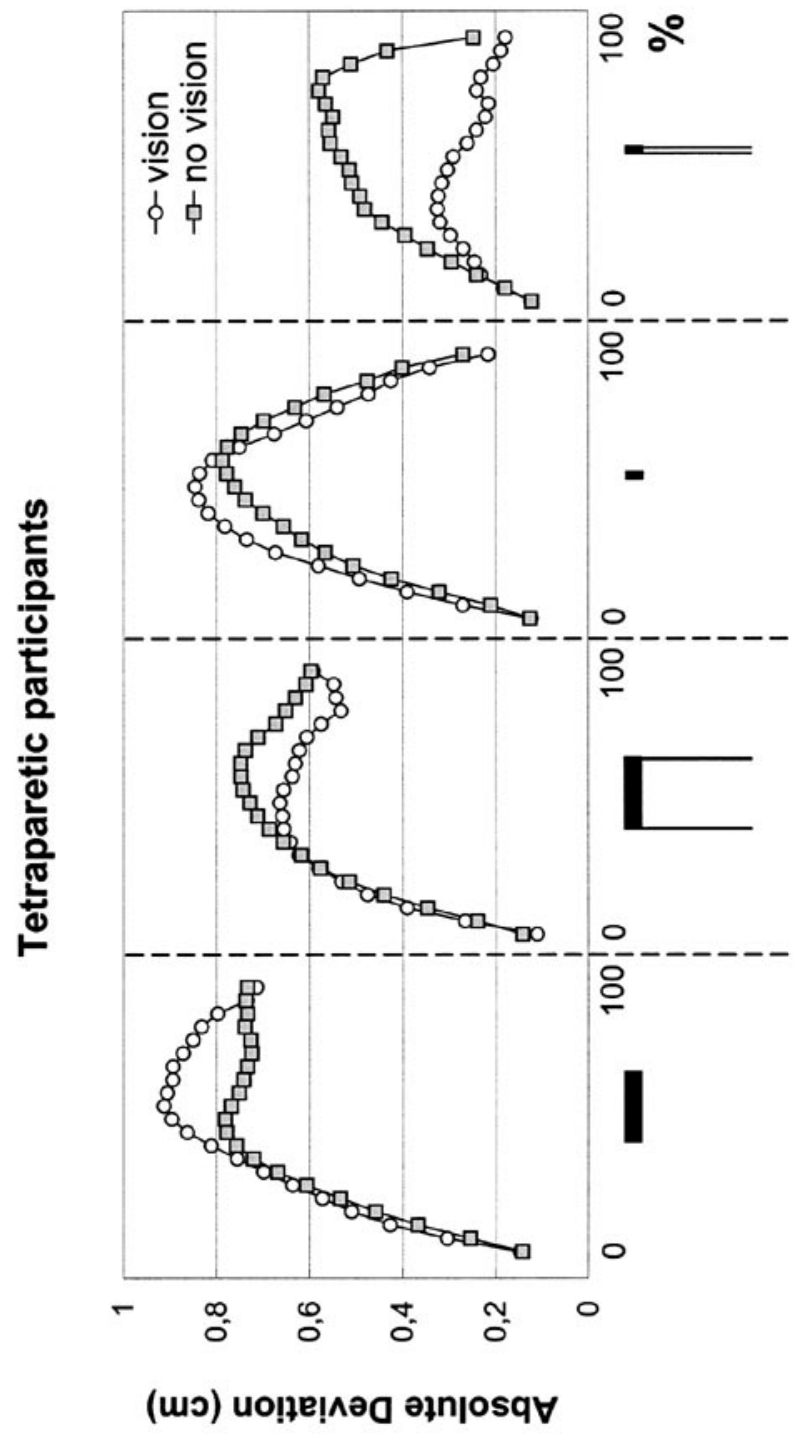

Figure 5-(continued) 
Percentage of MT Drawn Outside the Lines. No significant difference was found between the groups with regard to the percentage of MT that the pen was outside the lines (see Figure 6), which constituted our second measure of accuracy. When the distance between the lines measured $56 \mathrm{~mm}$, both groups remained between the lines in the vast majority of the trials; only two tetraparetic participants drew outside the lines in one and two trials, respectively. No effect of vision was present. When vision was blocked while participants had to move between two lines that were $5 \mathrm{~mm}$ apart (thus, very high accuracy demands), accuracy decreased, however, in both the tetraparetic group, $F(1,7)=54.14, p<.001$, and the control group, $F(1,7)=19.27, p=.003$. Contrary to our expectations, however, the drop in accuracy was similar in both groups. For both groups, the pen was outside of the lines during approximately $30 \%$ of the MT.

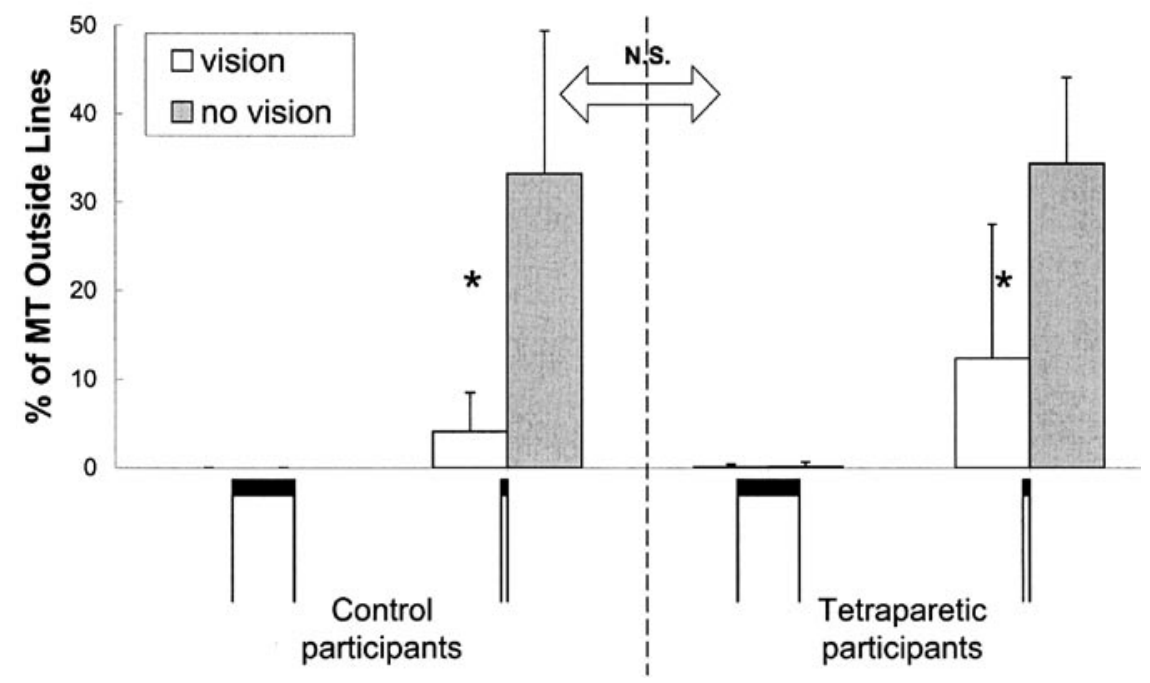

Figure 6-The percentage of movement time that control and tetraparetic participants drew outside of the lines: effects of target and vision. Asterisks represent significant effects of vision. Error bars represent between-participants variability (standard deviations). N.S.: not significant.

\section{Axial Pen Force}

The mean force that tetraparetic participants exerted during line drawing was 3.19 $\mathrm{N}$, while the mean force of controls was $1.23 \mathrm{~N}, t(14)=3.34, p=.003$. For the tetraparetic group, no effects of the manipulated task variables on pen force were found. It appeared, however, that the controls used, on average, $0.14 \mathrm{~N}$ more pen force when vision of the moving limb was blocked than when vision was available, $F(1,7)=8.17, p=.024$. Interestingly, for the tetraparetic group, a high positive correlation $(r=.94, p<.01)$ was found between the mean axial pen force and the mean absolute error made by the preferred hand in the passive proprioception test; the larger the mean absolute error, the higher the mean force. 


\section{Movement Time}

As expected, movement times were longer in the tetraparetic participants as compared to the controls, $t(14)=3.31, p=.003$ (see Figure 7). In the control group, a target $\times$ lines interaction was found, $F(1,7)=12.16, p=.010$. Step-down analyses showed that target width had an effect both with and without lines in this group ( $p=.008$ and $p=.010$, respectively). When the target was small, MTs were longer. The presence of lines only resulted in longer MTs when the target was small (thus, the distance between the lines was small; $p=.011)$. Blocking vision of the moving limb had no effect on the MTs of controls. A similar target $\times$ lines interaction was found for the tetraparetic group. A target $\times$ lines $\times$ vision interaction was, however, also found for that group, $F(1,7)=10.08, p=.016$. Blocking vision resulted in longer MTs in almost all combinations of target and lines $(p<.05)$, except for the most difficult combination: small target and lines present. In that condition, seven out of the eight tetraparetic participants had longer MTs when vision was available. This, however, was only a marginal trend $(p=.075)$, probably due to the large inter-participant variability.

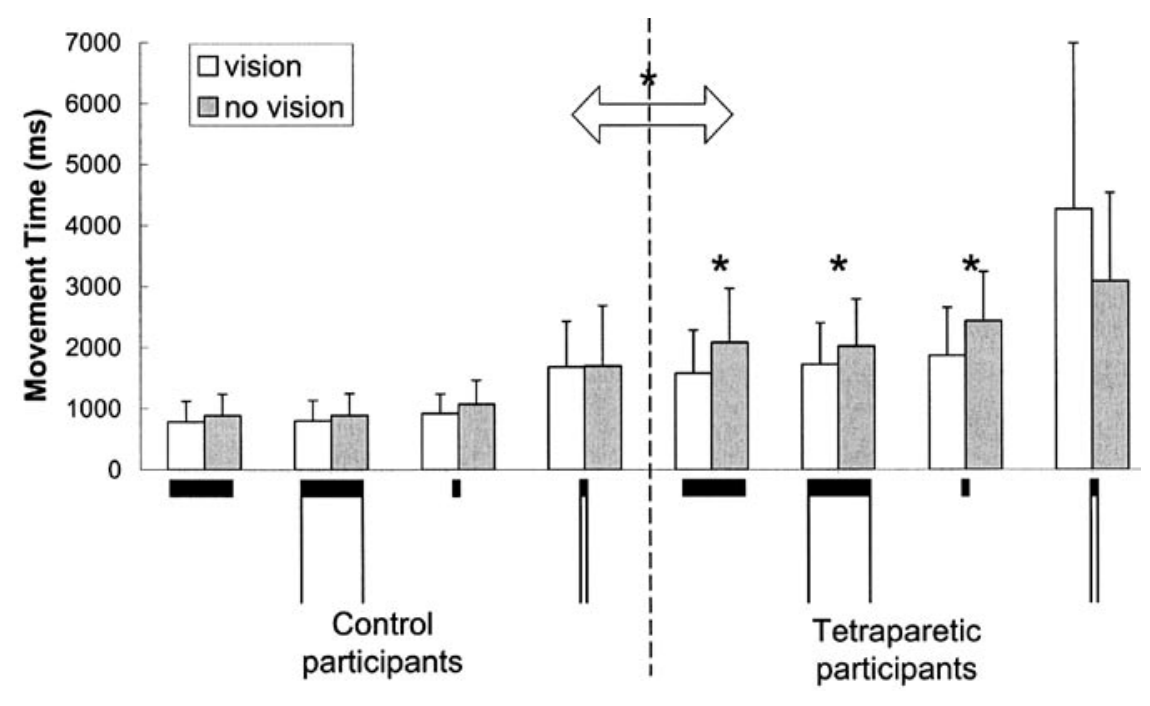

Figure 7-Movement times (ms) for the control and tetraparetic participants as a function of target, lines, and vision. Asterisks represent significant effects of vision or group, and error bars represent between-participants variability (standard deviations).

\section{Dysfluency}

Movements were performed less fluently in the tetraparetic group as compared to the control group, $t(14)=4.95, p<.001$ (see Figure 8). In both groups, a target $\times$ lines $\times$ vision interaction was found (control group: $F(1,7)=15.43, p=.006$; tetraparetic group: $F(1,7)=9.53, p=.018$ ). In the control group, the dysfluency increased when vision was blocked, but only when the target was large $(p<.05)$. The effect was reversed in the most difficult task condition (small target - lines 


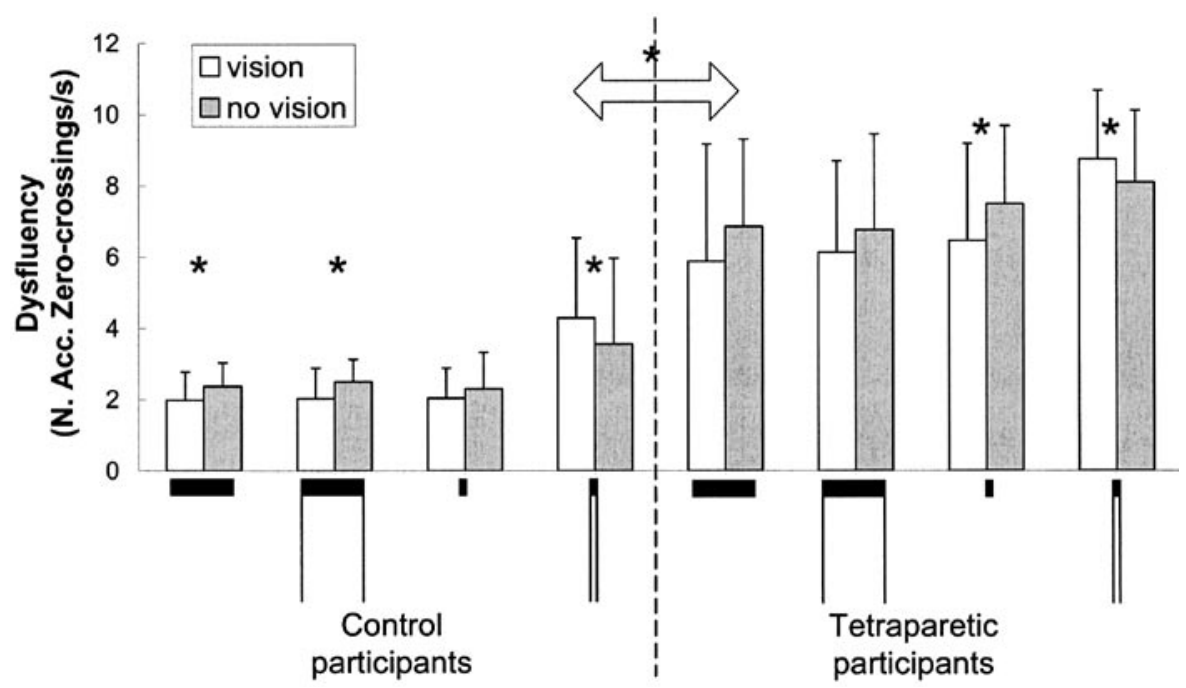

Figure 8-Dysfluency, expressed as the number of zero-crossings per second in the acceleration profile, for the control and tetraparetic participants as a function of target, lines, and vision. Asterisks represent significant effects of vision or group, and error bars represent between-participants variability (standard deviations).

present). Here, dysfluency was larger when vision was available $(p=.035)$. In the tetraparetic group, significant effects of vision were only found for the conditions in which the target was small; when a free movement was allowed (lines absent), blocking vision of the moving limb led to an increased dysfluency $(p=.025)$. When lines were present, however, blocking vision led to a decreased dysfluency ( $p=$ $.027)$, as was also the case in the control group.

\section{Correlations Between Proprioception and Performance in the Main Experiment}

In Table 2, individual data of the tetraparetic participants are shown: results of the active and passive proprioception test (mean absolute errors and variabilities of the error) and results (means and coefficients of variation [CV]) for the most complex condition of the main experiment (i.e., small target - lines present - no vision). We chose to show only the results for that specific condition because we wanted to explore the relationship between the measures of proprioception and performance in the main experiment. In this condition, the effects of an impaired proprioception would become most evident, since the accuracy constraints were very high and vision could not be used to make corrections.

First, some significant correlations were found with the absolute error scores in the active proprioception test, although it must be noted that the correlation coefficients only reached the significance level when we did not include participant number 7. Participants with a higher absolute error score in the active proprioception test had larger maximal lateral deviations $(r=+.89, p=.004$, one-tailed) and 
van Roon et al.

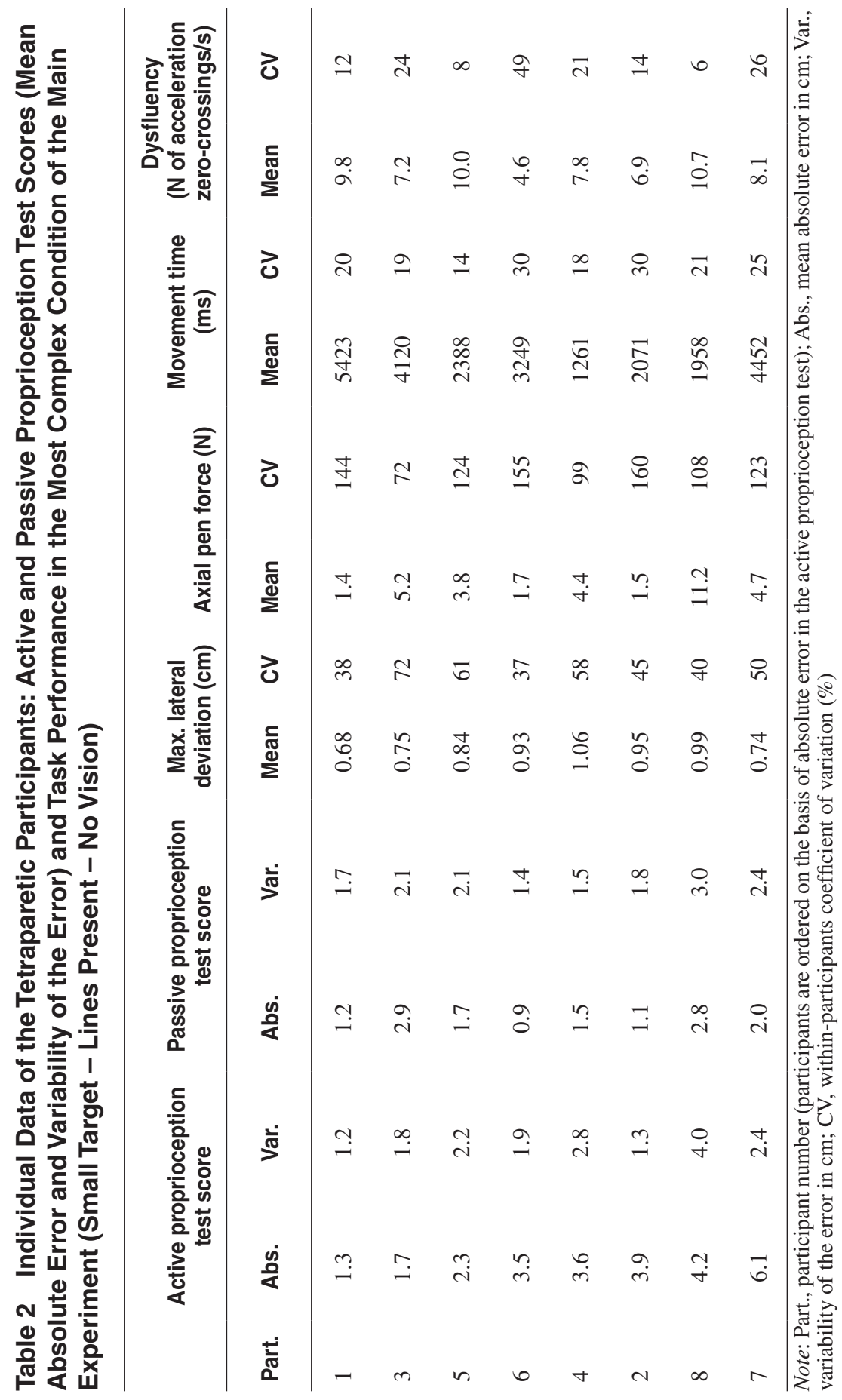


shorter MTs ( $r=-.86, p=.014$, two-tailed) in the most complex condition of the main experiment. It was also found, however, that tetraparetic participants with shorter MTs had higher maximal lateral deviations ( $r=+.98, p<.001$, one-tailed). Hence, participants who used less time to perform the drawing task moved less accurately. To determine whether the decreased accuracy was mainly a result of the decreased MT, we calculated the correlation between the absolute error score in the active proprioception test and the maximal lateral deviation with MT partialled out. The results showed that the correlation remained significant $(r=+.76$, $p=.04$, one-tailed).

Second, it was found that participants with higher absolute error scores in the passive proprioception test showed a larger variability (indicated by larger CVs) for the maximal lateral deviation $(r=+.64, p=.043$, one-tailed).

Finally, mean axial pen force in the most complex condition appeared to be higher in participants with higher absolute error scores in the passive proprioception test ( $r=+.86, p=.007$, one-tailed), in participants with a higher variability of the error in the passive proprioception test $(r=+.64, p=.043$, one-tailed), and in participants with a higher variability of the error in the active proprioception test ( $r=+.74, p=.019$, one-tailed).

\section{Discussion}

The aim of the present experiment was twofold: first, to examine whether people with $\mathrm{CP}$ really need visual information of the moving limb to be able to move accurately, and second, to examine strategies they might use to compensate for the loss of visual information. The two potential compensatory strategies tested here were increasing pen force and making slower movements.

Surprisingly, movement accuracy decreased to the same extent in both groups when vision of the moving limb was blocked. In addition, neither the tetraparetic participants nor the controls showed extreme sideward drift, despite the fact that movement corrections based on visual information were made impossible. This unexpected result could either point to a smaller need of the tetraparetic participants to visually monitor their movements than presumed (i.e., they were well able to pre-plan the movement), or to the use of strategies to compensate for the lack of visual information. Two possible strategies were examined in the present study, namely increasing pen force and slowing down movements. They will be discussed separately next.

It was shown that the overall axial pen force was higher in the tetraparetic group as compared to the control group. The participants with tetraparesis, however, did not increase pen force when vision was blocked, as was the case for the healthy participants. Furthermore, no effects of accuracy constraints were found. Thus, increasing pen force appeared to be a more general strategy in $\mathrm{CP}$, possibly to filter out unwanted noise from the motor system (Van Galen \& Van Huygevoort, 2000; Van Gemmert \& Van Galen, 1997) and/or to enhance proprioceptive input (Reinders-Messelink et al., 2001). This latter suggestion was supported by the finding of a high correlation between the mean pen force and the absolute error in the passive proprioception test for the tetraparetic participants. Thus, tetraparetic participants whose proprioception was more impaired used more pen force. 
The second possible compensatory strategy examined was a slowing down of the complete movement, that is, an increase in movement time. It was found that in the conditions in which visual feedback could not be used to correct the movement, the CP participants slowed down their movements (viz., increased MTs). This might be a strategy to decrease the noisiness of the motor command (Harris \& Wolpert, 1998) and/or to lengthen the time in which proprioceptive feedback can be exploited. The finding that the tetraparetic participants were able to diminish the lateral deviation after it had reached a maximum in conditions in which vision was blocked indicates that they might have used proprioceptive feedback then to correct the movement. In the most difficult movement condition (small target, lines present), the results were opposite, however. Here, the participants with CP tended to slow down when visual information of the moving limb was available. This finding suggests that under very high accuracy demands, visual guidance is used to perform the movement, as is also confirmed by the increased number of acceleration zero-crossings (viz., a decreased fluency of moving) in this condition. Blocking vision had no effect on MTs in controls, possibly because they are less dependent on visual feedback to move accurately as a result of better movement pre-planning and better proprioception. Indeed, recent results of Van Beers, Haggard, and Wolpert (2004) suggest that healthy people can correct their movements on the basis of proprioceptive feedback, and that these corrections become effective around $250 \mathrm{~ms}$ after movement onset.

The use of these compensatory mechanisms might also explain why we did not find larger absolute errors in our active proprioception test in the tetraparetic participants, although their passive proprioception appeared to be impaired. In the active proprioception test, participants were allowed to choose their preferred pen force and movement speed. Consequently, they might have been able to compensate for the lack of visual information. In contrast, in the passive proprioception test, employing these strategies was not possible as the force exerted to the ball and the speed of the movement were determined by the experimenter. This might have led to the proprioceptive impairment becoming more overt in this test condition.

To place the findings in perspective, some limitations of the present study merit discussion. The main and unexpected finding in the present study was the high accuracy of moving in the tetraparetic participants even when vision of the moving limb was blocked. Besides the explanations proposed earlier, we want to mention some other possible explanations. First, the 10 trials within a condition were performed in succession and movement extent and direction remained constant throughout the experiment. Participants could have formulated an internal model of the movement that could be used across trials, also in conditions in which vision of the moving limb was blocked. It has been proposed that the central nervous system uses sensory motor mappings called "internal models" to anticipate and adapt to dynamic environments (e.g., Shadmehr \& Mussa-Ivaldi, 1994). Previous studies on force generation during precision grip in children and adolescents with CP (e.g., Duff \& Gordon, 2003; Gordon \& Duff, 1999; Steenbergen et al., 1998) suggest that these individuals can use such an internal model of the object characteristics in an anticipatory manner for force scaling. As the basic movement in the present experiment (moving forward with the pen) did not vary among the different conditions, it might well be that control was based on an internal model that was already formed after a few trials. This model could have promoted accuracy of movement and, contrary to our expectations, could have led to a decreased need 
for visual information. In addition to this, more than 40 years ago, Fleishman and Rich (1963) showed that learning a novel motor task involves a shift in control from the visual to the proprioceptive system. Although the present task was new for the participants, all had experience with writing, so that they might be able to shift quickly to the proprioceptive "control mode," especially since the movement remained constant throughout the experiment.

Second, the drawing movements in this study had to be performed in a twodimensional workspace, i.e., on a digitizing tablet. The hand-pen system was continuously in contact with the surface of the digitizer, which led to extra proprioceptive input. It is possible that movement accuracy would have suffered more from the lack of visual information of the moving limb when movements should have had to be performed in a three-dimensional workspace.

Third, although vision of the hand during the movement was blocked in half of the trials, participants could always see the position of their hand before they started the movement (see also Desmurget, Rossetti, Jordan, Meckler, \& Prablanc, 1997; Ghez, Gordon, \& Ghilardi, 1995; Rossetti, Stelmach, Desmurget, Prablanc, \& Jeannerod, 1994). In healthy people, lower aiming errors were found when participants were instructed to foveate the starting base and then the target prior to movement initiation, but only when no dynamic visual information was available during the movement (Bédard \& Proteau, 2001). It was suggested that with such a procedure an eye-based representation of the movement is formed that can be used by the CNS to plan the aiming movement. In the present experiment, this could have led to the relatively small effect of removing visual information on accuracy of moving. Therefore, further research should examine if having this information at the start of the movement (initial position of the hand and configuration of the upper limb) is indeed essential to enable people with CP to move accurately.

Fourth, and finally, all tetraparetic participants were able to write with their least affected hand and the impairment of this hand was relatively mild. To add to the generality of the present results, the performance of individuals with $\mathrm{CP}$ who are more severely impaired should be studied as well. It is possible that movement accuracy is more affected by blocking vision in those individuals.

In sum, despite the inherent limitations, the present findings suggest that participants with mild tetraparetic CP do not depend on visual guidance to make accurate upper limb movements, despite the many (clinical) observations showing their increased reliance on visual guidance of the moving limb. While these participants might have adapted to their relatively unpredictable movement system by relying on visual guidance, the present study showed that removing the possibility of using this guidance was not detrimental to movement accuracy. Although clear differences between the tetraparetic group and the control group were found (longer MTs, more dysfluent movements, and a higher pen force), the present study demonstrates that people with CP can successfully compensate for a loss of visual information by lengthening the movement time.

\section{Acknowledgments}

This research was supported by a grant awarded by The Netherlands Organization for Scientific Research (NWO) to the second author for the research project "Adaptation in movement disorder." 


\section{References}

Abahnini, K., \& Proteau, L. (1999). The role of peripheral and central visual information for the directional control of manual aiming movements. Canadian Journal of Experimental Psychology, 53, 160-174.

Abrams, R.A., Meyer, D.E., \& Kornblum, S. (1990). Eye-hand coordination: oculomotor control in rapid aimed limb movements. Journal of Experimental Psychology: Human Perception and Performance, 16, 248-267.

Adamovich, S.V., Berkinblit, M.B., Hening, B., Sage, J., \& Poizner, H. (2001). The interaction of visual and proprioceptive inputs in pointing to actual and remembered targets in Parkinson's disease. Neuroscience, 104, 1027-1041.

Barnes, M., McLellan, L., \& Sutton, R. (1994). Spasticity. In R.J. Greenwood, M.P. Barnes, T.M. McMillan, \& C.D. Ward (Eds.), Neurological rehabilitation (pp. 161-172). Edinburgh: Churchill Livingstone.

Bédard, P., \& Proteau, L. (2001). On the role of static and dynamic visual afferent information in goal-directed aiming movements. Experimental Brain Research, 138, 419-431.

Bédard, P., \& Proteau, L. (2003). On the role of peripheral visual afferent information for the control of rapid video-aiming movements. Acta Psychologica, 113, 99-117.

Bédard, P., \& Proteau, L. (2004). On-line vs. off-line utilization of peripheral visual afferent information to ensure spatial accuracy of goal-directed movements. Experimental Brain Research, 158, 75-85.

Bekkering, H., Adam, J.J., Van den Aarssen, A., Kingma, H., \& Whiting, H.T. (1995). Interference between saccadic eye and goal-directed hand movements. Experimental Brain Research, 106, 475-484.

Berthier, N.E., Clifton, R.K., Gullapalli, V., McCall, D.D., \& Robin, D.J. (1996). Visual information and object size in the control of reaching. Journal of Motor Behavior, 28, 187-197.

Brown, J.V., Schumacher, U., Rohlmann, A., Ettlinger, G., Schmidt, R.C., \& Skreczek, W. (1989). Aimed movements to visual targets in hemiplegic and normal children: Is the "good" hand of children with infantile hemiplegia also normal? Neuropsychologia, 27, 283-302.

Churchill, A., Hopkins, B., Ronnqvist, L., \& Vogt, S. (2000). The role of visual information about hand position and environmental context in reaching and grasping. Experimental Brain Research, 134, 81-89.

Cody, F.W.J., Lovgreen, B., \& Schady, W. (1993). Increased dependence upon visual information of movement performance during visuo-motor tracking in cerebellar disorders. Electroencephalography and Clinical Neurophysiology, 89, 399-407.

Cooper, J., Majnemer, A., Rosenblatt, B., \& Birnbaum, R. (1995). The determination of sensory deficits in children with hemiplegic cerebral palsy. Journal of Child Neurology, 10, 300-309.

Desmurget, M., Rossetti, Y., Jordan, M., Meckler, C., \& Prablanc, C. (1997).Viewing the hand prior to movement improves accuracy of pointing performed toward the unseen contralateral hand. Experimental Brain Research, 115, 180-186.

Duff, S.V., \& Gordon, A.M. (2003). Learning of grasp control in children with hemiplegic cerebral palsy. Developmental Medicine and Child Neurology, 45, 746-757.

Eliasson, A., Rösblad, B., \& Forssberg, H. (2004). Disturbances in programming goaldirected arm movements in children with ADHD. Developmental Medicine and Child Neurology, 46, 19-27.

Filloux, F.M. (1996). Neuropathophysiology of movement disorders in cerebral palsy. Journal of Child Neurology, 11, S5-S12.

Fleishman, E.A., \& Rich, S. (1963). Role of kinesthetic and spatial-visual abilities in perceptual-motor learning. Journal of Experimental Psychology, 66, 6-11. 
Ghez, C., Gordon, J., \& Ghilardi, M.F. (1995). Impairments of reaching movements in patients without proprioception. II. Effects of visual information on accuracy. Journal of Neurophysiology, 73, 361-372.

Gordon, A.M., \& Duff, S.V. (1999). Fingertip forces during object manipulation in children with hemiplegic cerebral palsy. I: Anticipatory scaling. Developmental Medicine and Child Neurology, 41, 166-175.

Gribble, P.L., Mullin, L.I., Cothros, N., \& Mattar, A. (2003). Role of cocontraction in arm movement accuracy. Journal of Neurophysiology, 89, 2396-2405.

Harris, C.M., \& Wolpert, D.M. (1998). Signal-dependent noise determines motor planning. Nature, 394, 780-784.

Khan, M.A., Lawrence, G.P., Franks, I.M., \& Buckolz, E. (2004). The utilization of visual feedback from peripheral and central vision in the control of direction. Experimental Brain Research, 158, 241-251.

Klockgether, T., \& Dichgans, J. (1994). Visual control of arm movement in Parkinson's Disease. Movement Disorders, 9, 48-56.

Lance, J.W. (1980). Symposium synopsis. In R.G. Feldman, R.R. Young, \& W.P. Koella, Spasticity: Disordered motor control (pp. 485-494). Chicago: Yearbook Medical.

Mathiowetz, V., Volland, G., Kashman, N., \& Weber, K. (1985). Adult norms for the Box and Block Test of manual dexterity. American Journal of Occupational Therapy, 39, 386-391.

Neggers, S.F.W., \& Bekkering, H. (1999). Integration of visual and somatosensory target information in goal-directed eye and arm movements. Experimental Brain Research, 125, 97-107.

Opila-Lehman, J., Short, M.A., \& Trombly, C.A. (1985). Kinesthetic recall of children with athetoid and spastic cerebral palsy and of non-handicapped children. Developmental Medicine and Child Neurology, 27, 223-230.

Pryde, K.M., Roy, E.A., Bryden, P.J., Kalbfleisch, L., \& MacKinnon, G.E. (1998). Goal-directed movement control in a case of hemiparesis. Brain and Cognition, 37, 122-124.

Reinders-Messelink, H.A., Schoemaker, M.M., Snijders, T A.B., Göeken, L.N.H., Bökkerink, J.P.M., \& Kamps, W.A. (2001). Analysis of handwriting of children during treatment for acute lymphoblastic leukemia. Medical and Pediatric Oncology, 37, 393-399.

Rossetti, Y., Stelmach, G.E., Desmurget, M., Prablanc, C., \& Jeannerod, M. (1994). The effect of viewing the static hand prior to movement onset on pointing kinematics and accuracy. Experimental Brain Research, 101, 323-330.

Shadmehr, R., \& Mussa-Ivaldi, F.A. (1994). Adaptive representation of dynamics during learning of a motor task. Journal of Neuroscience, 14, 3208-3224.

Smyth, M.M., Peacock, K.A., \& Katamba, J. (2004). The role of sight of the hand in the development of prehension in childhood. The Quarterly Journal of Experimental Psychology, 57A, 269-296.

Steenbergen, B. (2000). The planning and coordination of prehension movements in spastic hemiparesis. Doctoral dissertation, University of Nijmegen, The Netherlands.

Steenbergen, B., Hulstijn, W., Lemmens, I.H.L., \& Meulenbroek, R.G.J. (1998). The timing of prehensile movements in subjects with cerebral palsy. Developmental Medicine and Child Neurology, 40, 108-114.

Steenbergen, B., Meulenbroek, R.G.J., \& Rosenbaum, D.A. (2004). Constraints on grip selection in hemiparetic cerebral palsy: effects of lesional side, end-point accuracy, and context. Cognitive Brain Research, 19, 145-159.

Steenbergen, B., \& Van der Kamp, J. (2004). Control of prehension in hemiparetic cerebral palsy: similarities and differences between the ipsi- and contra-lesional sides of the body. Developmental Medicine and Child Neurology, 46, 325-332.

Trombly, C.A. (1992). Deficits of reaching in subjects with left hemiparesis: a pilot study. The American Journal of Occupational Therapy, 46, 887-897. 
Van Beers, R.J., Baraduc, P., \& Wolpert, D.M. (2002). Role of uncertainty in sensorimotor control. Philosophical Transactions of the Royal Society of London Series B-Biological sciences, 357, 1137-1145.

Van Beers, R.J., Haggard, P., \& Wolpert, D.M. (2004). The role of execution noise in movement variability. Journal of Neurophysiology, 91, 1050-1063.

Van Galen, G.P., \& Van Huygevoort, M. (2000). Error, stress and the role of neuromotor noise in space oriented behaviour. Biological Psychology, 51, 151-171.

Van Gemmert, A.W.A., \& Van Galen, G.P. (1997). Stress, neuromotor noise, and human performance: a theoretical perspective. Journal of Experimental Psychology: Human Perception and Performance, 23, 1299-1313.

Van Thiel, E., Meulenbroek, R.G.J., Hulstijn, W., \& Steenbergen, B. (2000). Kinematics of fast hemiparetic aiming movements towards stationary and moving targets. Experimental Brain Research, 132, 230-242.

Vercher, J.L., Magenes, G., Prablanc, C., \& Gauthier, G.M. (1994). Eye-head-hand coordination in pointing at visual targets: spatial and temporal analysis. Experimental Brain Research, 99, 507-523.

Wann, J.P. (1991). The integrity of visual-proprioceptive mapping in cerebral palsy. Neuropsychologia, 29, 1095-1106. 